\title{
ANÁLISIS COSTE-EFICACIA DE LAS COMBINACIONES FIJAS DE PROSTAGLANDINAS/PROSTAMIDA PARA EL TRATAMIENTO DEL GLAUCOMA
}

\author{
COST-EFFICACY ANALYSIS OF FIXED COMBINATIONS OF \\ PROSTAGLANDIN/PROSTAMIDE FOR TREATING GLAUCOMA
}

\author{
MARTÍNEZ A ${ }^{1}$, SLOF J $^{2}$
}

\section{RESUMEN}

Objetivo: Evaluar la relación coste-eficacia de las tres combinaciones fijas de prostaglandinas/prostamida con timolol actualmente disponibles en el mercado español [bimatoprost con timolol (BT) Ganfort $^{\circledR}$, latanoprost con timolol (LT) - Xalacom ${ }^{\circledR}$ y travoprost con timolol (TT) - DuoTrav ${ }^{\circledR}$ ].

Métodos: Dado que no existen estudios que comparen de una manera directa la eficacia de estos fármacos, se llevó a cabo una revisión sistemática de la evidencia indirecta en lengua inglesa. Se estimaron el consumo de recursos sanitarios y su coste a partir de un modelo esquemático de la práctica clínica habitual en nuestro medio. Se calcularon para cada fármaco la relación coste-eficacia media y la relación coste-eficacia incremental, en términos de euros por punto porcentual de reducción de presión intraocular (PIO) en un periodo de tres meses.

Resultados: BT redujo la PIO en un $35,1 \%$, LT en un $35,0 \%$ y TT en un $34,7 \%$. El coste-eficacia medio se estimó para BT en 5,34 € por punto porcentual de reducción de PIO, para LT en 5,40 €, y para TT en 5,45 €. El coste-eficacia incremental (coste adicional por punto porcentual adicional de
Objective: To assess the cost-efficacy of three fixed-combination glaucoma treatments currently available in Spain [bimatoprost with timolol (BT)Ganfort $^{\circledR}$, latanoprost with timolol (LT)- Xalacom ${ }^{\circledR}$, and travoprost with timolol (TT)- DuoTrav $\left.{ }^{\circledR}\right]$.

Methods: Because no studies are available that give a direct comparison of these drugs, a systematic review was carried out to assess their efficacy. Resource consumption and costs were estimated using a model of usual local practice. For each of the three drugs, average and incremental cost-efficacy ratios were determined in terms of euros per percentage point of reduction of intraocular pressure (IOP) over a three-month period.

Results: BT reduced IOP by $35.1 \%$, LT by $35.0 \%$ and TT by $34.7 \%$. Average cost-efficacy was estimated to be $€ 5.34$ per percentage point of IOP reduction with $\mathrm{BT}$, $€ 5.40$ with $\mathrm{LT}$, and $€ 5.45$ with TT. Incremental cost-efficacy (incremental cost per incremental percentage point of IOP reduction) was estimated to be $€ 94.65$ for LT vs. TT, and was negative for BT vs. TT and BT vs. LT, since in both cases BT was more efficacious and less expensive.

\footnotetext{
Recibido: 28/9/07. Aceptado: 29/9/08.

1 Licenciado en Medicina. Instituto Gallego de Oftalmología. Santiago de Compostela. La Coruña. España.

2 Doctor en Ciencias Económicas y Empresariales. Universidad Autónoma de Barcelona. Bellaterra. Barcelona. España.

El estudio en que se basa este artículo ha recibido financiación de los laboratorios Allergan.

Correspondencia:

John Slof

Departamento de Economía de la Empresa

Universidad Autónoma de Barcelona

08193 Bellaterra (Barcelona)

España

E-mail: ericjohn.slof@uab.es
} 
reducción de PIO) se estimó para LT frente a TT en 94,65 €, y resultó negativo para BT frente a TT y BT frente a LT, debido a que BT en ambos casos era a la vez más eficaz y más económico.

Conclusiones: Bimatoprost/timolol parece ser la alternativa más económica comparada con travoprost/timolol y latanoprost/timolol, a igual o mayor eficacia y tolerabilidad.

Palabras clave: Glaucoma, análisis de costes, betabloqueante, análogo de prostaglandinas, combinaciones fijas.
Conclusions: Compared to travoprost/timolol and latanoprost/timolol, bimatoprost/timolol appears to be the most economic alternative, with equal or better efficacy and safety results (Arch Soc Esp Oftalmol 2008; 83: 595-600).

Key words: Glaucoma, cost analysis, beta-blocker, prostaglandin analogue, fixed combinations.

\section{INTRODUCCIÓN}

El glaucoma primario de ángulo abierto (GPAA), la forma más común del glaucoma, es una neuropatía óptica crónica y progresiva que supone una de las principales causas de ceguera en el mundo occidental. Se caracteriza por una pérdida progresiva de fibras nerviosas de la retina que puede ir asociada con una elevación indolora de la presión intraocular (PIO) y una pérdida de campo visual. Si no se trata adecuadamente, el curso natural de esta patología puede desembocar en una ceguera irreversible (1).

Aunque las estimaciones en la literatura de la prevalencia de glaucoma oscilan considerablemente, no hay duda de que se trata de un problema que afecta a un número muy elevado de personas, sobre todo de edad avanzada, y que representa una carga elevada (2,3). Las estimaciones en estudios internacionales varían entre $0,4 \%$ y $8,8 \%$ de la población mayor de 40 años, mientras en España se ha situado la prevalencia entorno del $2 \%$ (4-6).

La etiología exacta del GPAA es desconocida, pero se sabe que un aumento de la PIO es el factor de riesgo más importante y por el momento, el único sobre el que se puede actuar. Distintos estudios han demostrado tanto en glaucomas con tensión elevada, tensión normal, y en hipertensos oculares un beneficio con el descenso de la PIO (7-10). Por este motivo, el control de la PIO es el objetivo principal de los tratamientos del glaucoma. El tratamiento médico es actualmente la primera línea de actuación para alcanzar la PIO objetivo en pacientes con GPAA (11). Los análogos de las prostaglandinas/prostamida y los beta-bloqueantes son los fármacos de primera línea, quedando los inhibidores de la anhidrasa y alfa-agonistas como fármacos de segunda línea.
No obstante, estudios recientes han sugerido que la combinación de algunos de estos fármacos aporta beneficios superiores a las combinaciones no fijas. Particularmente, los beta-bloqueantes y análogos de prostaglandinas tienen mecanismos de acción complementarios, y últimamente, gotas que combinan estas terapias están ganando popularidad (12-15).

Las combinaciones fijas mejoran la eficacia hipotensora, disminuyen los efectos secundarios, y permiten pautas más cómodas con el consiguiente mejor cumplimiento por parte de los pacientes, pero también tienen un coste superior. Dada la cada vez más importante limitación de recursos del Sistema Nacional de Salud, se hacen necesarios estudios de coste-eficacia para estos tratamientos. El presente estudio pretende evaluar la relación coste-eficacia de las tres combinaciones fijas de prostaglandinas/prostamida con timolol disponibles en el mercado español.

\section{SUJETOS, MATERIAL Y MÉTODOS}

Las combinaciones fijas de beta-bloqueante y análogos prostanoides actualmente disponibles en España son bimatoprost $0,03 \%$ con timolol $0,50 \%$, (BT) (Ganfort $^{\circledR}$, Allergan, Irvine, CA), latanoprost $0,005 \%$ con timolol 0,50\%, (LT) (Xalacom ${ }^{\circledR}$, Pharmacia, Kalamazoo, MI, and Pfizer, New York, NY), y travoprost $0,004 \%$ con timolol $0,50 \%$, (TT) (DuoTrav ${ }^{\circledR}$, Alcon, Forth Worth, TX). Dado que no existen estudios que comparen de una manera directa la eficacia de estos fármacos, se llevó a cabo una revisión sistemática de la evidencia indirecta en lengua inglesa.

Los datos sobre BT fueron obtenidos de dos estudios recientes con un total de 1.061 pacientes [(Katz 
LJ, Lewis RA, Batoosingh AL, Liu C, for the Ganfort $^{\circledR}$ Investigators' Group II. Bimatoprost/Timolol Fixed Combination: A One-Year, Double-Masked, Randomized Parallel Comparison to Its Individual Components in Patients With Glaucoma or Ocular Hypertension. ARVO 2007) y (Brandt JD, Cantor LB, Batoosingh AL, Liu C for the Ganfort ${ }^{\circledR}$ Investigators' Group. A 3-Month, Randomized Study Comparing Bimatoprost/Timolol Fixed-Combination Therapy to Monotherapy With Bimatoprost or Timolol in Patients With Glaucoma or Ocular Hypertension. IGS 2007)]. En estos estudios 533 pacientes fueron tratados con BT, y los demás con sus componentes individuales. La revisión sistemática identificó cuatro estudios comparables con LT y TT (24-27), con datos de un total de 1.484 pacientes (tabla I). Los criterios de búsqueda fueron: ensayo controlado doble ciego, mismo horizonte temporal que en los ensayos con $\mathrm{BT}$, misma variable de eficacia, periodo de lavado antes de la visita inicial, y edad y valores basales comparables. El indicador de eficacia primaria común en los ensayos incluidos era la diferencia entre la PIO con tratamiento y la PIO basal, ambas medidas en la mañana, conseguida a lo largo de un periodo de tres meses. Como la PIO basal no era idéntica en los diferentes estudios, se calculó para cada caso la disminución porcentual, y fue ésta la variable de eficacia utilizada en la determinación del coste-eficacia.

A partir de un modelo esquemático de la práctica clínica (fig. 1), se determinaron los costes asociados al tratamiento con los antiglaucomatosos y se calcularon para cada fármaco la relación coste-eficacia media y la relación coste-eficacia incremental. A estos efectos, se definió la relación coste-eficacia media como el resultado de dividir el coste del tratamiento con un fármaco por su eficacia hipotensora. La relación coste-eficacia incremental fue definida como el resultado de dividir los costes adicio-

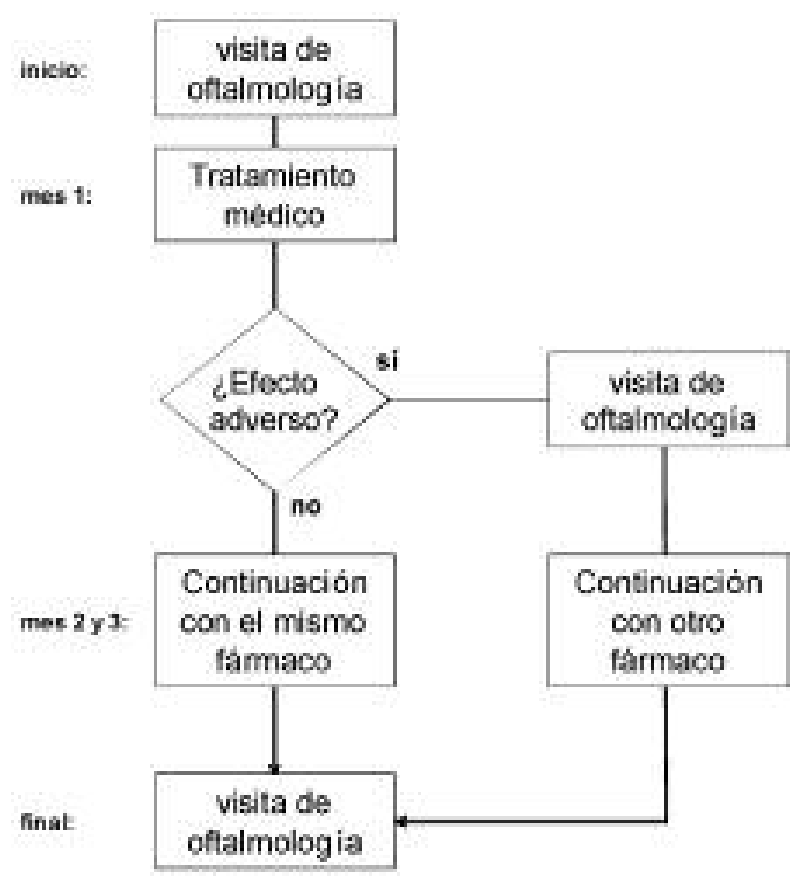

Fig. 1: Modelo esquemático de la práctica clínica.

nales del tratamiento con un fármaco respecto al comparador más económico por la eficacia adicional frente a este mismo comparador.

$$
\begin{gathered}
\text { Coste-eficacia media de tto. } \mathrm{A}=\frac{\text { coste de tto. A }}{\text { eficacia de tto. A }} \\
\begin{array}{c}
\text { Coste-eficacia incremental } \\
\text { de tto. A vs. B }
\end{array}
\end{gathered}=\frac{\text { coste adicional de tto. A vs. B }}{\text { eficacia adicional de A vs. B }}
$$

\begin{tabular}{|c|c|c|c|c|c|c|}
\hline Fármaco estudiado & Fuente del estudio & $\mathrm{N}$ & $\begin{array}{l}\text { Edad media } \\
\text { de pacientes } \\
\quad(\text { años })\end{array}$ & $\begin{array}{l}\text { PIO basal } \\
(\mathrm{mm} \mathrm{Hg})\end{array}$ & $\begin{array}{l}\text { Reducción } \\
\text { de PIO } \\
\text { a los } 3 \text { meses }\end{array}$ & $\begin{array}{l}\text { Abandono por } \\
\text { acontecimiento adverso } \\
\text { relacionado }\end{array}$ \\
\hline Bimatoprost con timolol & $\begin{array}{l}\text { Katz et al } 2007 \text { (16) y } \\
\text { Brandt et al } 2007 \text { (17) }\end{array}$ & 533 & 62,1 & 25,9 & $35,1 \%$ & $3,6 \%$ \\
\hline Travoprost con timolol & $\begin{array}{l}\text { Hughes et al } 2005(26) \\
\text { Barneby et al } 2005(27) \\
\text { Schuman et al } 2005(28) \\
\text { Conjunto }\end{array}$ & $\begin{array}{r}151 \\
82 \\
155 \\
388\end{array}$ & $\begin{array}{l}64,2 \\
63,0 \\
62,4\end{array}$ & $\begin{array}{l}25,3 \\
30,2 \\
25,6 \\
26,5\end{array}$ & $\begin{array}{l}34,4 \% \\
38,1 \% \\
32,9 \% \\
34,6 \%\end{array}$ & $\begin{array}{c}2,65 \% \\
\text { No informó sobre AA } \\
5,2 \% \\
3,92 \%\end{array}$ \\
\hline Latanoprost con timolol & Diestelhorst et al 2006 (29) & 255 & 61,8 & 26,0 & $35,0 \%$ & $2,3 \%$ \\
\hline
\end{tabular}

Se estimaron el consumo de recursos sanitarios y su coste, de acuerdo con la práctica habitual en nuestro medio (tabla II). Se supuso que sería nece-

Tabla I. Análisis conjunto de ensayos clínicos 
Tabla II. Supuestos sobre el consumo y coste de recursos sanitarios

\begin{tabular}{|c|c|}
\hline Parámetro & Supuesto \\
\hline Pauta de medicación & Un frasco al mes \\
\hline Coste de los fármacos & $\begin{array}{l}\text { PVP más IVA de la presentación } \\
\text { más económica: } \\
\text { - Ganfort }{ }^{\circledR} 3 \mathrm{ml}: 23,21 € \\
\text { - DuoTrav }{ }^{\circledR} 2,5 \mathrm{ml}: 23,42 € \\
\text { - Xalacom }{ }^{\circledR} 2,5 \mathrm{ml}: 23,88 €\end{array}$ \\
\hline Visitas médicas & $\begin{array}{l}2 \text { visitas de oftalmología por perío- } \\
\text { do de } 3 \text { meses }\end{array}$ \\
\hline Coste de visitas médicas & $58 €$ por visita \\
\hline Efectos adversos & $\begin{array}{l}1 \text { visita de oftalmología adicional; } \\
\text { cambio a medicación alternativa }\end{array}$ \\
\hline
\end{tabular}

sario adquirir un frasco de cada medicamento al mes, dado que no se recomienda el uso de estos fármacos después de que haya pasado este tiempo desde la apertura del frasco. Además se supuso que en un periodo de tres meses cada paciente haría como mínimo dos visitas médicas. También se consideró que se haría una visita adicional en el caso de que se produjera un acontecimiento adverso que hiciera aconsejable un cambio de tratamiento. La incidencia de esta situación se obtuvo a partir de los casos observados durante los ensayos clínicos con cada fármaco. Por último, se asumió que los pacientes afectados continuarían su tratamiento con uno de los dos otros comparadores a partir del segundo mes del modelo.

El consumo de los recursos sanitarios se valoró en términos monetarios desde una perspectiva social. Concretamente, para valorar el consumo de los fármacos, se utilizó su precio de venta al público más el impuesto sobre el valor añadido, es decir, el precio en oficina de farmacia, sin tener en cuenta ningún tipo de descuento por ser beneficiario de la Seguridad Social. El coste de las visitas médicas, por otro lado, se obtuvo de la contabilidad analítica del Instituto
Galego de Oftalmoloxía, que se consideraba representativo para la sanidad pública española.

\section{RESULTADOS}

Se resumen los resultados obtenidos en la tabla III. BT redujo la PIO en un $35,1 \%$ en un periodo de tres meses; TT y LT, en cambio, redujeron en el mismo periodo la PIO en un $34,6 \%$ y $35,0 \%$, respectivamente. La ratio coste-eficacia media se estimó para BT en 5,34 € por punto porcentual de reducción de la PIO, para LT en 5,40 €, y para TT en $5,45 €$. La ratio coste-eficacia incremental, es decir el coste adicional por punto porcentual adicional de reducción de PIO, se estimó para LT vs. TT en 94,65 €. El coste-eficacia incremental de BT vs. TT y el de BT vs. LT resultaron negativos, debido a que BT en ambos casos fue más eficaz y más económico, presentándose por tanto, como la opción «dominante».

\section{DISCUSIÓN}

Actualmente, los beta-bloqueantes y los análogos de prostaglandina/prostamida son las opciones de primera línea para la reducción de la PIO en pacientes con GPAA. La evidencia clínica más reciente indica que los tratamientos combinados de estos fármacos pueden constituir una alternativa eficaz e igualmente segura. No obstante, pautas complejas que incluyan la administración de varios fármacos, pueden llevar a un peor cumplimiento del tratamiento del GPAA, lo que se considera una de las principales causas del fracaso del mismo (16). Las combinaciones fijas constituyen, por tanto, una buena alternativa.

En un análisis económico de fármacos antiglaucomatosos administrados en monoterapia desarro-

Tabla III. Resumen de resultados

$\begin{array}{ccc}\begin{array}{c}\text { Eficacia } \\ \text { (reducción } \\ \text { de PIO) }\end{array} & \begin{array}{c}\text { Coste del tratamiento } \\ \text { durante tres meses }\end{array} & \begin{array}{c}\text { Ratio de coste- } \\ \text { eficacia media } \\ \end{array} \\ & (€) & (€ \text { por punto } \\ & & \text { porcentual de } \\ \text { educción de PIO) }\end{array}$
Ratio de coste-eficacia incremental (€ adicional por punto porcentual de reducción de $\mathrm{PIO}$ adicional)
vs. vs. vs. bimatoprost travoprost latanoprost con timolol con timolol con timolol

\begin{tabular}{|c|c|c|c|c|c|c|}
\hline Bimatoprost con timolol & $35,1 \%$ & 187,75 & 5,34 & - & Dominante & Dominante \\
\hline Travoprost con timolol & $34,6 \%$ & 188,54 & 5,45 & Dominado & - & 94,65 \\
\hline Latanoprost con timolol & $35,0 \%$ & 188,95 & 5,40 & Dominado & 94,65 & - \\
\hline
\end{tabular}


llado en España, Galindo et al (17) observaron, al igual que otros estudios internacionales (18-22), que el grupo de beta-bloqueantes presentaba una eficacia hipotensora menor que los análogos de las prostaglandinas, pero dado su bajo precio, su ratio coste-eficacia hipotensora media (en euros por punto de porcentaje de reducción de PIO) era más bajo. La eficacia encontrada en nuestro estudio para los productos combinados está por encima de los valores hallados por Galindo et al para las monoterapias, mientras nuestras ratios de coste-eficacia media se sitúan entre los valores de Galindo et al para los beta-bloqueantes y análogos de prostaglandinas en monoterapia. A pesar de que las metodologías utilizadas no son del todo equiparables, esto sugiere que los productos combinados no sólo son la alternativa más eficaz, sino también que la mayor eficacia frente a los beta-bloqueantes se obtiene a un coste por punto porcentual de reducción de PIO relativamente más bajo que con los análogos de prostaglandinas en monoterapia.

No obstante, el objetivo principal de nuestro estudio fue comparar entre sí las combinaciones fijas con prostaglandinas disponibles en España, y no comparar las monoterapias con las terapias combinadas. En este sentido, nuestros resultados indican que, aunque las diferencias entre los comparadores no son muy grandes, BT domina, desde el punto de vista farmacoeconómico, a TT y LT. Es decir, BT se presenta como la opción más eficaz y menos cara de las tres analizadas. Según una publicación reciente que sigue una metodología coincidente con la nuestra, se obtienen resultados muy parecidos en el contexto de otros sistemas sanitarios europeos (28).

Sin embargo, hay que señalar que nuestro trabajo tiene varias limitaciones. En primer lugar, no nos basamos en una comparación directa de la eficacia de BT, TT y LT, sino en un meta-análisis ad-hoc. En ausencia de ensayos clínicos de comparación directa, una revisión exhaustiva de la literatura y la comparación indirecta de alternativas farmacológicas pueden ser una alternativa razonable. No obstante, hay que señalar que en los estudios encontrados los valores basales de PIO fueron diferentes en los diversos grupos, lo cual hace que idealmente los grupos no sean comparables. Otra limitación es la falta de intervalos de confianza para los resultados conjuntos, debido a la dificultad de calcular éstos a partir de la información revelada en las fuentes utilizadas. Por ello, no se puede descartar que las diferencias en la eficacia observada se deban al azar. No obstante, la conclusión de que desde una perspectiva económica, BT representa la alternativa dominante de las tres analizadas, no se vería alterada en el caso de que su eficacia o tolerabilidad no fueran estadísticamente superiores a sus comparadores, ya que seguiría siendo la opción de menor coste.

Respecto a la eficacia, aunque la reducción en la PIO está considerada como una variable adecuada para valorar la eficacia de los tratamientos para el GPAA, la relación entre la reducción de la PIO y el progreso del GPAA no está clara. No fue posible valorar la eficacia de los tratamientos en la variable de resultados que en última instancia realmente es relevante, es decir, la preservación de la calidad de vida del paciente (23), pero evidentemente, esta limitación también lo fue de los ensayos originales en que se basa nuestro estudio.

Finalmente, al igual que en otros estudios similares $(17,18)$, no se han tenido en cuenta los costes que se derivan de la falta de eficacia más allá del horizonte temporal del modelo utilizado, como por ejemplo, los de una cirugía posterior. Tampoco se consideraron los costes indirectos asociados a la patología y su tratamiento. Se puede señalar que esta falta introduce un sesgo contra el tratamiento más eficaz, pero no altera las conclusiones ya que afecta con el mismo signo a todos los comparadores.

En conclusión, la combinación de dos fármacos de primera línea en una presentación única es eficaz en el tratamiento del GPAA y representa una opción terapéutica valiosa que puede ayudar a mejorar el cumplimiento. A pesar de las limitaciones de nuestro estudio, los resultados del mismo indican que de las tres combinaciones fijas de prostaglandinas/prostamida con timolol actualmente disponibles en el mercado español, BT parece ser la alternativa más económica, a igual o mayor eficacia y tolerabilidad.

\section{AGRADECIMIENTOS}

Agradecemos los valiosos comentarios y sugerencias recibidos del Dr. Antonio Ferreras.

\section{BIBLIOGRAFÍA}

1. Van Buskirk EM, Cioffi GA. Glaucomatous optic neuropathy. Am J Ophthalmol 1992; 113: 447-452.

2. Traverso CE, Walt JG, Kelly SP, Hommer AH, Bron AM, Denis $P$, et al. Direct costs of glaucoma and severity of the disease: a multinational long term study of resource utilisation in Europe. Br J Ophthalmol 2005; 89:1245-1249. 
3. Rouland JF, Berdeaux G, Lafuma A. The economic burden of glaucoma and ocular hypertension: implications for patient management: a review. Drugs Aging 2005; 22 : 315-321.

4. Gimeno A, Jiménez $R$, Ferrer JL, Rinaldi T, Suárez $O$, Fernández $G$, et al. Planificación y resultados de un programa de prevención del glaucoma. Rev San Hig Pub 1988; 63: 1571-1582.

5. Zafra Pérez JJ, Villegas Pérez MP, Canteras Jordana M, Miralles De Imperial J. Presión intraocular y prevalencia de glaucoma oculto en una población de la región de Murcia. Arch Soc Esp Oftalmol 2000; 75: 171-178.

6. Jaén Días J, Sanz Alcolea I, López De Castro F, Pérez Martínez T, Ortega Campos P, Corral Morales R. Glaucoma e hipertensión ocular en atención primaria. Aten Primaria 2001; 28: 23-30.

7. The effectiveness of intraocular pressure reduction in the treatment of normal-tension glaucoma. Collaborative Normal Tension Glaucoma Study Group. Am J Ophthalmol 1998; 126: 498-505.

8. The Advanced Glaucoma Intervention Study (AGIS): 7. The relationship between control of intraocular pressure and visual field deterioration. The AGIS Investigators. Am J Ophthalmol 2000; 130: 429-440.

9. Kass MA, Heuer DK, Higginbotham EJ, Johnson CA, Keltner JL, Miller JP, et al. The Ocular Hypertension Treatment Study: a randomized trial determines that topical ocular hypotensive medication delays or prevents the onset of primary open-angle glaucoma. Arch Ophthalmol 2002; 120: 701-713.

10. Leske MC, Heijl A, Hussein M, Bengtsson B, Hyman L, Honaroff E, et al. Factors for glaucoma progression and the effect of treatment: the early manifest glaucoma trial. Arch Ophthalmol 2003; 121: 48-56.

11. European Glaucoma Society. Terminology and Guidelines for Glaucoma, IInd edition. 2003

12. Choudhri S, Wand M, Shields MB. A comparison of dorzolamide-timolol combination versus the concomitant drugs. Am J Ophthalmol. 2000; 130:832-833.

13. Denis $P$. Intérêt des traitements par combinaisons fixes. $J$ Fr Ophtalmol 2006; 29: 2S45-2S48.

14. Khouri AS, Realini T, Fechtner RD. Use of fixed-dose combination drugs for the treatment of glaucoma. Drugs Aging 2007; 24: 1007-1016.

15. Hommer A, Ganfort Investigators Group I. A double-masked, randomized, parallel comparison of a fixed combination of bimatoprost $0.03 \% /$ timolol $0.5 \%$ with non-fixed combination use in patients with glaucoma or ocular hypertension. Eur J Ophthalmol 2007; 17: 53-62.
16. Shaw ME. Increasing compliance with glaucoma therapy: «so, convince me I have something wrong with my eyes». Insight 2005; 30: 7-9.

17. Galindo-Ferreiro A, Sánchez-Tocino, Fernández-Muñoz M, Iglesias-Cortiñas D. Análisis coste-eficicacia de los fármacos antiglaumatosos más utilizados. Arch Soc Esp Oftalmol 2004; 79: 379-384.

18. Kobelt G, Jönsson L. Modeling cost of treatment with new topical treatments for glaucoma. Results from France and the United Kingdom. Int J Technol Assess Health Care 1999; 15: 207-219.

19. Fiscella RG. Costs of glaucoma medications. Am J Health Syst Pharm 1998; 55: 272-275.

20. Marchetti A, Magar R, An P, Nichol M. Clinical and economic impact of new trends in glaucoma treatment. MedGenMed 2001; 3: 6.

21. Vold SD, Wiggins DA, Jackimiec J. Cost analysis of glaucoma medications. J Glaucoma 2000; 9: 150-153.

22. Ikeda H, Sato E, Kitaura T, Fukuchi H, Kimura Y, Kihira $K$. Daily cost of ophthalmic solutions for treating glaucoma in Japan. Jpn J Ophthalmol 2001; 45: 99-102.

23. Muñóz Negrete FJ. Evidencias sobre presión intraocular objetivo. Arch Soc Esp Oftalmol 2002; 11: 587-588.

24. Hughes BA, Bacharach J, Craven ER, Kaback MB, Mallick $S$, Landry TA, et al. A three-month, multicenter, doublemasked study of the safety and efficacy of travoprost $0.004 \% /$ timolol $0.5 \%$ ophthalmic solution compared to travoprost $0.004 \%$ ophthalmic solution and timolol $0.5 \%$ dosed concomitantly in subjects with open angle glaucoma or ocular hypertension. J Glaucoma 2005; 14: 392-399.

25. Barnebey HS, Orgengo-Nania S, Flowers BE, Samples J, Mallick S, Landry TA, et al. The safety and efficacy of travoprost $0.004 \% /$ timolol $0.5 \%$ fixed combination ophthalmic solution. Am J Ophthalmol 2005; 140: 1-7.

26. Schuman JS, Katz GJ, Lewis RA, Henry JC, Mallick S, Wells DT, et al. Efficacy and safety of a fixed combination of travoprost $0.004 \% /$ timolol $0.5 \%$ ophthalmic solution once daily for open-angle glaucoma or ocular hypertension. Am J Ophthalmol 2005; 140: 242-250.

27. Diestelhorst $M$, Larsson L; European-Canadian Latanoprost Fixed Combination Study Group. A 12-week, randomized, double-masked, multicenter study of the fixed combination of latanoprost and timolol in the evening versus the individual components. Ophthalmology 2006; 113: 70-76.

28. Hommer A, Wickstrøm J, Friis MM, Steeds C, Thygesen J, Ferreras A, et al. A cost-effectiveness analysis of fixedcombination therapies in patients with open-angle glaucoma: a European perspective. Curr Med Res Opin 2008; 23: 1057-1063. 